\title{
Percepções de estudantes de enfermagem sobre práticas educativas em imunização de adultos com o uso de Simulação Clínica
}

\author{
Nursing students' perceptions about adult immunization educational practices \\ using Clinical Simulation
}

Raphael Raniere de Oliveira Costa $\left(\mathbb{D}{ }^{1} \triangle\right.$, Soraya Maria de Medeiros $\mathbb{D}^{2}{ }^{2}$, Verónica Rita Dias Coutinho $\mathbb{D}^{3}$, Rodrigo Guimarães dos Santos Almeida ${ }^{(\mathbb{D}}$, Marília Souto de Araújo ${ }^{2}{ }^{2}$

1 Universidade Federal do Rio Grande do Norte - UFRN, Escola Multicampi de Ciências Médicas do Rio Grande do Norte - EMCM

Caicó, Rio Grande do Norte, Brasil.

2 Universidade Federal do Rio Grande do Norte - UFRN, Departamento de Enfermagem. Natal, Rio Grande do Norte, Brasil.

${ }^{3}$ Escola Superior de Enfermagem de Coimbra. Coimbra, Portugal.

${ }^{4}$ Universidade Federal de Mato Grosso do Sul - UFMS. Campo Grande, Mato Grosso do Sul, Brasil.

Como citar este artigo (How to cite this article):

Costa RRO, Medeiros SM, Coutinho VRD, Almeida RGS, Araújo MS. Percepções de estudantes de enfermagem sobre práticas educativas em imunização de adultos com o uso de Simulação Clínica (Nursing students' perceptions about adult immunization educational practices using Clinical Simulation). Sci Med. 2019;29(3):e34267. https://doi.org/10.15448/1980-6108.2019.3.34267

\section{RESUMO}

OBJETIVO: Identificar e comparar as percepções de estudantes sobre práticas educativas no ensino de imunização de adultos.

MÉTODO: Estudo observacional, descritivo, comparativo, realizado com estudantes de enfermagem. Estes foram alocados em um grupo de estratégias tradicionais (exposição dialogada e simulação clínica para treino de habilidades) e grupo que se associava a simulação clínica de alta-fidelidade. Após receberem as intervenções, foi aplicada a versão portuguesa do Educational Practices Questionnaire.

RESULTADOS: O estudo foi realizado com 34 estudantes de enfermagem. O grupo em que foi associada a simulação clínica de alta-fidelidade apresentou médias superiores em sete dos oito domínios das subescalas de concordância e importância, com significância estatística na subescala de importância, no domínio "maneiras diferentes de aprendizagem" $(\mathrm{p}=0,018)$.

CONCLUSÃO: A estratégia da simulação clínica de alta-fidelidade pontua os requisitos de uma boa prática educacional na medida em que proporciona uma aprendizagem ativa, colaborativa, diferente das estratégias tradicionais e por proporcionar expectativas positivas aos estudantes.

UNITERMOS: Simulação; ensino de enfermagem; atenção primária a saúde; estudantes de enfermagem.

\section{ABSTRACT}

AIMS: To identify and compare the perceptions of students about educational practices in the teaching of immunization of adults.

METHODS: Observational, descriptive, comparative, performed with nursing students. These students were allocated to two groups: traditional strategies group (dialogue and clinical simulation for skills training) and group associated with high fidelity clinical simulation. After receiving the interventions, the Portuguese version of the Educational Practices Questionnaire was applied.

RESULTS: The group associated with high fidelity clinical simulation. presented higher means in seven of the eight domains of concordance and importance subscales, with statistical significance in the subscale of importance in the "different ways of learning" domain ( $\mathrm{p}=0.018$ ).

CONCLUSION: The high-fidelity clinical simulation strategy scores the requirements of good educational practice in that it provides active, collaborative learning, different from traditional strategies, and provides positive expectations for students.

KEYWORDS: Simulation; nursing education; primary health care; nursing students. 


\section{INTRODUÇÃO}

$\mathrm{Na}$ área da saúde, a instrumentalização nos aspectos técnicos, éticos e políticos para a transformação dos processos de trabalho se apresenta como uma necessidade real. Essa transformação busca superar a ideia da fragmentação do cuidado. Para tanto, adotam-se as novas formas de ensinar e aprender, a integração entre teoria e prática, a articulação entre ensino e serviços, a busca pela imersão em diversos conhecimentos e a articulação com outras profissões da saúde. Busca-se a capacidade de reflexão e a transformação da realidade social $[1,2]$.

Apesar dessas inúmeras mudanças no contexto político, econômico, tecnológico e educacional, a forma como se ensina na enfermagem continua quase que inalterada. $\mathrm{O}$ modelo tradicional continua a ser o mais seguido nas escolas de enfermagem. É comum observar um instrutor clínico levando um grupo de estudantes para uma unidade de saúde onde cada aluno recebe um ou dois pacientes para cuidar. As atividades desempenhadas ficam limitadas ao 4tempo do instrutor para acompanhar e observar procedimentos, e ao acaso das necessidades do paciente designado ao estudante [3].

Sabe-se que as práticas pedagógicas adotadas pelo docente contribuem para o desenvolvimento da capacidade crítica e reflexiva do estudante, além de identificar as potencialidades e fragilidades para o aprender e guiá-los para atingir os objetivos da aprendizagem [4]. No contexto das práticas pedagógicas, as estratégias de ensino e aprendizagem são apresentadas como ferramentas que o docente utiliza para atingir os objetivos de aprendizagem. Entre estas, podemos citar a aula expositiva e dialogada, a simulação clínica de baixa fidelidade, e a simulação de alta fidelidade.

A aula expositiva e dialogada é uma proposta alternativa as palestras tradicionais dos docentes. Nessa estratégia, o estudante tem a possibilidade de interagir durante a exposição dos conteúdos. Essa interação possibilita a mobilidade e pode desembocar em um clima favorável à aprendizagem. Nesses momentos, é indispensável considerar o conhecimento prévio dos estudantes. Deve-se favorecer uma análise crítica e produção de novos conhecimentos [5].

Outra estratégia de ensino e aprendizagem utilizada no ensino de enfermagem é a simulação clínica de baixa fidelidade para o treino de habilidades. A partir dessa estratégia podem-se repetir inúmeras vezes sequências de técnicas, procedimentos, condutas e outros elementos pertinentes à formação do enfer- meiro [5]. Nessa perspectiva, trabalha-se competências e habilidades com menor nível de complexidade.

Já na simulação clínica de alta fidelidade, as competências e habilidades trabalhadas assumem um caráter mais complexo. Para tanto, o uso de ferramentas - como simuladores mais robustos e atores treinados para executar o papel de usuário - o que pode contribuir para aumentar a fidelidade psicológica dos cenários simulados, aumentando o realismo (grau em que um cenário se aproxima da realidade) [6].

A partir da simulação clínica de alta fidelidade, os estudantes podem adquirir competências e habilidades relacionadas à tomada de decisão, comunicação e ofertar assistência ao paciente simulado vivenciando os aspectos diretos e indiretos que estão relacionados a uma determinada situação $[7,8]$.

Dada as inúmeras possibilidades que a estratégia da simulação clínica (de baixa e alta fidelidade) pode oferecer no contexto da formação do profissional enfermeiro, encoraja-se a sua inserção no currículo das instituições formadoras para a aquisição de competências e habilidades pertinentes a tal [9].

Além de contemplar o estudante com diferentes formas, meios e estratégias de aprender, é imprescindível pensar numa reformulação curricular que permita o diálogo entre os saberes. Que proporcione o desenvolvimento de competências e as habilidades específicas e transversais, o desenvolvimento de métodos diferentes e eficazes de avaliação do processo e o investimento em infraestrutura e formação de recursos humanos [10].

Durante a formação do enfermeiro, é indispensável que se oportunize diferentes formas de ensinar e aprender. É fundamental que se avalie tais práticas quanto aos aspectos que permitem a aprendizagem. $\mathrm{Na}$ literatura, poucos estudos compararam os resultados de experiências simuladas associadas a experiências mais tradicionais de ensino e aprendizagem $[11,12]$.

Nesse ínterim, encoraja-se a utilização da simulação clínica em todo o processo formativo, não restringindo o seu uso à situações que envolvem o contexto hospitalar. Com isso, estudo multicêntrico recomenda a utilização de experiências clínicas simuladas no âmbito da atenção primária à saúde [13].

Sabe-se ainda que, no contexto da atenção primária a saúde brasileira, o profissional enfermeiro é responsável pelo gerenciamento e execução de práticas que oportunizam a melhoria dos indicadores de saúde relacionados a práticas de imunização. Ao considerar esse importante papel, se faz necessário qualificar estudantes de graduação em enfermagem para que estes possam, ao serem inseridos nos cenários de 
práticas que exijam essas competências e habilidades, contribuir para a melhoria e efetividade dos serviços e dessas práticas.

Nessa perspectiva, o estudo teve por objetivo comparar e identificar as percepções de estudantes sobre práticas educativas no ensino de imunização de adultos no contexto da graduação em enfermagem.

\section{MÉTODOS}

Estudo observacional, descritivo, comparativo, aprovado pelo Comitê de Ética em Pesquisa sob protocolo $\mathrm{n}$ - 1.958.827 e CAAE $\mathrm{n}$ o 64874817.3 .0000 .5537$. O estudo foi realizado em uma universidade pública federal brasileira no período de maio a junho de 2017.

Os estudantes do curso de graduação em enfermagem, compreendidos e matriculados entre o 5 o e $9^{\circ}$ semestre, foram convidados a participar do curso de extensão que deu origem a coleta dos dados. A escolha por estudantes matriculados entre os semestres anteriormente referidos se deu em detrimento da disponibilidade dos estudantes e ao considerar que, a partir desses semestres, eles têm a oportunidade de vivenciar tais práticas no contexto curricular do curso o qual estão vinculados.

O convite ao público-alvo da pesquisa foi realizado através de abordagem pessoal nas salas de aula e pela página eletrônica específica da instituição. Ao concordar em participar, os estudantes foram convidados a preencher uma ficha de inscrição para uma posterior aleatorização e designação a modalidades do curso os quais iriam ser vinculados.

Considerou-se as seguintes variáveis: sexo, idade, índice de rendimento acadêmico, experiência profissional na área de atenção primária à saúde e diagnóstico do sistema representacional preferencial.

No estudo, foram utilizados os seguintes critérios de inclusão: ser estudante da graduação em enfermagem regularmente matriculado entre o 5 o e 9 o semestre do curso; e ter o mínimo de $75 \%$ de frequência durante o curso oferecido. Foram excluídos os estudantes que não estavam presentes nos outros momentos de intervenção e aplicação dos instrumentos da pesquisa; os bolsistas e colaboradores que contribuíram para a execução do estudo.

A amostra foi do tipo não probabilística por conveniência e após a distribuição entre os grupos, os estudantes participaram de curso com 40 horas referente à imunização de adulto.

Comparou-se as seguintes estratégias de ensino e aprendizagem em dois grupos: grupo controle com estratégias tradicionais com aula expositiva e simulação clínica para treino de habilidades; e grupo experimental com aula expositiva, simulação clínica para treino de habilidades e simulação de alta fidelidade quanto aos seguintes aspectos: aprendizagem ativa, colaboração, maneiras diferentes de aprendizagem e altas expectativas.

Em ambos os grupos, as aulas expositivas e dialogadas foram construídas e conduzidas pelo pesquisador. Os conteúdos e o material de estudo foram enviados aos participantes previamente aos encontros em sala de aula. No total, a carga horária disponibilizada para estes momentos foi de oito horas.

A simulação clínica para o treino de habilidades aconteceu no laboratório da universidade e previamente a estas atividades, os estudantes receberam guias do tipo checklist. Foram montadas quatro estações no laboratório de enfermagem onde foram trabalhadas as habilidades. Na estação 1, o objetivo de aprendizagem foi realizar técnicas de administração de doses de imunobiológicos (vias intramuscular e subcutânea); na estação 2, o objetivo de aprendizagem era conhecer e identificar rotinas e organização da sala de vacina; na estação 3 , os estudantes realizaram práticas de aprazamentos de doses de vacinas; Já na estação 4, o objetivo de aprendizagem era a tomada de decisão referente a diversas situações vacinal de adultos.

$\mathrm{Na}$ oportunidade, os estudantes foram divididos em pequenos grupos - 4 a 5 estudantes - e se revezaram entre as estações. Após a consolidação das atividades em cada estação, os pesquisadores, um grupo de três docentes de enfermagem, forneceram um feedback para os grupos participantes. O tempo para a realização das tarefas, em cada estação, foi de 20 minutos.

Além das atividades anteriormente mencionadas, o grupo experimental participou de simulações clínicas de alta fidelidade. Para tanto, foram criados três cenários. Estes foram construídos a partir dos instrumentos e referenciais disponíveis na literatura a partir dos modelos do Hospital Universitário de Tübingen, da Alemanha, e o da Universidade Anhembi Morumbi, Brasil [14].

Para a criação de cenários, foi utilizada a estrutura S.M.A.R.T. (Specific, Measurable, Achievable, Realistic, Time-Phased) essa estrutura orienta a construção dos objetivos e o design de cenários em simulação e leva em consideração o objetivo específico do cenário, a forma de mensuração dos resultados a partir do objetivo, se este é alcançável e como alcançá-lo, se é realista e o tempo necessário para alcançá-lo [15]. Após esse procedimento, os cenários foram testados e validados por especialistas quanto 
a aparência e conteúdo. Os especialistas consultados foram os pesquisadores do projeto.

Foram criados três cenários. O cenário 1 contemplava um paciente acometido por evento traumático/ acidental no contexto da atenção primária à saúde. $\mathrm{O}$ cenário 2 abrangia a administração e aprazamento de imunobiológicos na atenção primária à saúde. Já o cenário 3 , abrangia situações de contraindicações da administração de imunobiológicos.

Os três cenários foram executados no mesmo dia, foi reservado o total de 50 minutos para cada cenário, sendo cinco minutos de reconhecimento do cenário; 15 minutos para o desenvolvimento, e 30 minutos de debriefing. Para esta atividade, foi ofertado um encontro presencial de quatro horas/aula. A sessão de feedback realizou-se o debriefing, seguido das etapas: descrição, análise e síntese.

Como recurso, foi utilizada a ferramenta pacientepadrão, que são atores treinados para atuar e reproduzir comportamentos de usuários em diversas situações e estabelecimentos de assistência à saúde [16]. Os atores foram convidados pelo pesquisador e treinados previamente pela equipe do projeto de pesquisa - voluntários, enfermeiros e docentes do ensino superior. Os atores foram calibrados a partir de reuniões, envolvendo-os juntamente com os pesquisadores, esclarecendo e definindo as formas de atuação e as falas.

Após o término do curso, todos os estudantes responderam um questionário de avaliação das atividades realizadas. Para tanto, foi aplicado o Questionário de Práticas Educativas (versão portuguesa do Educational Practices Questionnaire). Os pesquisadores optaram pela escolha deste questionário em razão da sua aplicabilidade junta estratégia pedagógica de simulação clínica.

O questionário foi desenvolvido pela National League for Nursing, que é uma organização dedicada a excelência no ensino de enfermagem, e validada para a versão portuguesa. $\mathrm{O}$ instrumento possui 16 itens distribuídos em quatro fatores, a saber: aprendizagem ativa, colaboração, maneiras diferentes de aprendizagem, e altas expectativas. Além disso, conta com duas subescalas: concordância e importância [17].

$\mathrm{Na}$ subescala de concordância, constam os seguintes padrões de respostas: 1 - discordo totalmente da afirmação, 2 - discordo da afirmação, 3 - indeciso (nem concordo nem discordo da afirmação), 4 - concordo com a afirmação, 5 - concordo totalmente com a afirmação, e não aplicável - a declaração não diz respeito à atividade realizada. Já na subescala de importância, consta os seguintes itens: 1 - não é importante, 2 - um pouco importante, 3 - neutro, 4 - im- portante, 5 - muito importante. No instrumento, o padrão de resposta é do tipo Likert [17].

Com o objetivo de estabelecer parâmetros de equivalência entre os grupos, realizou-se os seguintes testes estatísticos: teste do qui-quadrado, o teste exato de Fisher, o teste de Mann - Whitney, e o teste de Shapiro Wilks, todos para um nível de significância de 5\%. Para a análise da consistência interna, foi utilizado o teste alpha de Cronbach. Para a comparação dos grupos foi utilizado o teste U de Mann Whitney, o valor de Z, e o p-valor para um nível de significância de 5\%. Os dados foram analisados pelo SPSS versão 24 .

\section{RESULTADOS}

A amostra inicial foi de 58 estudantes que após serem aplicados os critérios de inclusão, a amostra final foi constituída de 34 estudantes, 17 em cada grupo.

$\mathrm{Na}$ análise de consistência interna, o questionário de práticas educativas apresentou um alpha de Cronbach de 0,8. O alpha da subescala de concordância foi de 0,735. Já na subescala de importância, o índice foi de 0,7. A Tabela 1 apresenta o perfil sociodemográfico dos participantes do estudo quanto ao sexo e faixa etária. A média de idade do grupo controle foi de 22,2 anos (mínima: 18 anos; máxima: 34 anos) e o do grupo experimental foi de 22,3 anos (mínima: 19 anos; máxima: 31 anos).

Tabela 1. Perfil sociodemográfico dos estudantes participantes do grupo controle e do grupo experimental quanto ao sexo e idade.

\begin{tabular}{lccc}
\hline & Total & $\begin{array}{c}\text { Grupo } \\
\text { controle }\end{array}$ & $\begin{array}{c}\text { Grupo } \\
\text { experimental }\end{array}$ \\
\hline $\mathrm{n}$ & 34 & 17 & 17 \\
Sexo feminino, $\mathrm{n}(\%)$ & $27(79,4)$ & 13 & 14 \\
Idade em anos, $\mathrm{n}(\%)$ & & & \\
$\quad 18-20$ & $5(14,7)$ & $4(23,5)$ & $1(5,9)$ \\
$21-23$ & $21(61,8)$ & $9(52,9)$ & $12(70,6)$ \\
$24-26$ & $6(17,6)$ & $3(17,6)$ & $3(17,6)$ \\
$>26$ & $2(5,9)$ & $1(5,9)$ & $1(5,9)$ \\
\hline
\end{tabular}

A Tabela 2 apresenta resultados referente ao questionário de práticas educativas, onde os estudantes informaram o grau de importância e de concordância sobre afirmações referente a aprendizagem ativa, colaboração, maneiras diferentes de aprendizagem e altas expectativas. Nesse sentido, tem-se na Tabela 2 a distribuição da média e do desvio padrão dos resultados das subescalas de práticas educativas. Dessa forma, em ambas as subescalas, em ambos os grupos, as médias foram superiores a 4,3 . 
Tabela 2. Pontuação global de concordância e importância das dimensões da escala de práticas educativas dos grupos controle e experimental.

\begin{tabular}{|c|c|c|c|c|}
\hline & \multicolumn{2}{|c|}{ Grupo controle $(n=17)$} & \multicolumn{2}{|c|}{ Grupo experimental $(n=17)$} \\
\hline & Concordância & Importância & Concordância & Importância \\
\hline Aprendizagem ativa & $4,3 \pm 0,3$ & $4,5 \pm 0,4$ & $4,5 \pm 0,3$ & $4,5 \pm 0,4$ \\
\hline Colaboração & $4,5 \pm 0,4$ & $4,6 \pm 0,4$ & $4,7 \pm 0,5$ & $4,7 \pm 0,4$ \\
\hline Maneiras diferentes de aprendizagem & $4,3 \pm 0,5$ & $4,5 \pm 0,5$ & $4,7 \pm 0,5$ & $4,6 \pm 0,5$ \\
\hline Altas expectativas & $4,5 \pm 0,6$ & $4,5 \pm 0,5$ & $4,5 \pm 0,6$ & $4,5 \pm 0,5$ \\
\hline
\end{tabular}

Valores expressos em média \pm desvio-padrão.

Nas subescalas de concordância e importância, o grupo experimental apresentou maiores escores médios em quase todos os domínios, exceto no domínio "expectativas" da subescala de concordância. Neste domínio, o grupo controle apresentou escore médio de $4,5 \pm 0,6$ enquanto estes valores no grupo experimental foram de 4,5 $\pm 0,6$.

A Tabela 3 trata da avaliação referente a satisfação dos estudantes e autoconfiança na aprendizagem, apresentando o posto médio das subescalas. Foram encontradas diferenças estatisticamente significativas na subescala de importância, especificamente no domínio "maneiras diferentes de aprendizagem" $(\mathrm{p}=0,018)$. Nessa perspectiva, é possível compreender que os estudantes reconhecem a importância da diversificação das maneiras de aprender e avaliar a aprendizagem.

É importante destacar também que no domínio "aprendizagem ativa", da subescala de concordância, houve uma diferença importante do posto médio entre os grupos experimental e controle $(20,1$ e 14,8, respectivamente). Além disso, é válido destacar que o grupo experimental apresentou postos médios superiores em sete, dos oito domínios das subescalas. Porém, não foi encontrado diferenças significância estatística nos demais domínios.

Tabela 3. Valores dos postos médios da satisfação com a aprendizagem atual e autoconfiança na aprendizagem dos estudantes dos grupos controle e experimental.

\begin{tabular}{lccc}
\hline Domínios & $\begin{array}{c}\text { Grupo } \\
\text { controle }\end{array}$ & $\begin{array}{c}\text { Grupo } \\
\text { experimental }\end{array}$ & P \\
Concordância & 14,8 & 20,1 & 0,122 \\
$\quad$ Aprendizagem ativa & 14,8 & 20,1 & 0,122 \\
Colaboração & 13,5 & 21,5 & 0,018 \\
$\quad$ Maneiras diferentes de aprendizagem & 17,4 & 17,5 & 0,973 \\
Altas expectativas & & & \\
Importância & 16,3 & 18,6 & 0,518 \\
$\quad$ Aprendizagem ativa & 15,9 & 19,0 & 0,375 \\
Colaboração & 16,2 & 18,7 & 0,474 \\
$\quad$ Maneiras diferentes de aprendizagem & 17,9 & 17,0 & 0,812 \\
$\quad$ Altas expectativas & & & \\
\hline
\end{tabular}

Tabela 4. Valores médios da concordância e importância das dimensões do questionário de práticas educativas dos grupos controle e experimental.

\begin{tabular}{|c|c|c|c|c|}
\hline \multirow[b]{2}{*}{ Domínios/Variáveis } & \multicolumn{2}{|c|}{ Subescala de concordância } & \multicolumn{2}{|c|}{ Subescala de importância } \\
\hline & $\begin{array}{l}\text { Grupo } \\
\text { controle }\end{array}$ & $\begin{array}{c}\text { Grupo } \\
\text { experimental }\end{array}$ & $\begin{array}{l}\text { Grupo } \\
\text { controle }\end{array}$ & $\begin{array}{l}\text { Grupo } \\
\text { experimental }\end{array}$ \\
\hline \multicolumn{5}{|l|}{ Aprendizagem ativa } \\
\hline $\begin{array}{l}\text { 1. Durante a atividade de simulação eu tive a oportunidade de discutir as ideias e os conceitos } \\
\text { ensinados no curso com o professor e outros alunos. }\end{array}$ & $4,6 \pm 0,4$ & $4,5 \pm 0,6$ & $4,70 \pm 0,46$ & $4,64 \pm 0,7$ \\
\hline 2. Eu participei ativamente da sessão de debriefing após a simulação. & $3,8 \pm 1,0$ & $4,3 \pm 0,7$ & $4,76 \pm 0,4$ & $4,5 \pm 0,6$ \\
\hline 3. Eu tive a oportunidade de refletir mais sobre meus comentários durante a sessão de debriefing. & $4,0 \pm 0,8$ & $4,4 \pm 0,6$ & $4,5 \pm 0,6$ & $4,4 \pm 0,6$ \\
\hline 4. Houve oportunidade suficiente na simulação para descobrir se eu compreendi claramente o material. & $4,1 \pm 0,7$ & $4,6 \pm 0,4$ & $4,5 \pm 0,5$ & $4,7 \pm 0,4$ \\
\hline 5. Eu aprendi com os comentários feitos pelo professor antes, durante ou após a simulação. & $4,7 \pm 0,4$ & $4,8 \pm 0,3$ & $4,5 \pm 0,6$ & $4,7 \pm 0,4$ \\
\hline 6. Eu recebi pistas durante a simulação, em tempo oportuno. & $4,0 \pm 0,7$ & $3,8 \pm 1,3$ & $4,0 \pm 0,7$ & $4,0 \pm 0,8$ \\
\hline 7. Eu tive a oportunidade de discutir os objetivos da simulação com o meu professor. & $3,9 \pm 0,8$ & $4,3 \pm 0,8$ & $4,2 \pm 0,7$ & $4,5 \pm 0,8$ \\
\hline 8. Eu tive a oportunidade de discutir ideias e conceitos ensinados na simulação com o meu professor. & $4,2 \pm 0,7$ & $4,8 \pm 0,39$ & $4,5 \pm 0,5$ & $4,6 \pm 0,6$ \\
\hline 9. O professor foi capaz de responder às necessidades individuais dos alunos durante a simulação. & $4,7 \pm 0,4$ & $4,7 \pm 0,5$ & $4,7 \pm 0,4$ & $4,7 \pm 0,4$ \\
\hline 10. O uso de atividades de simulação tornou meu tempo de aprendizagem mais produtivo. & $4,7 \pm 0,4$ & $4,7 \pm 0,4$ & $4,7 \pm 0,4$ & $4,7 \pm 0,4$ \\
\hline \multicolumn{5}{|l|}{ Colaboração } \\
\hline 11. Eu tive a oportunidade de trabalhar com meus colegas durante a simulação. & $4,5 \pm 0,6$ & $4,8 \pm 0,3$ & $4,5 \pm 0,6$ & $4,7 \pm 0,4$ \\
\hline 12. Durante a simulação, os meus colegas e eu tivemos de trabalhar na situação clínica juntos. & $4,5 \pm 0,5$ & $4,6 \pm 0,9$ & $4,7 \pm 0,4$ & $4,7 \pm 0,5$ \\
\hline \multicolumn{5}{|l|}{ Maneiras diferentes de aprendizagem } \\
\hline 13. A simulação ofereceu várias maneiras para aprender o material & $4,3 \pm 0,7$ & $4,8 \pm 0,5$ & $4,5 \pm 0,6$ & $4,7 \pm 0,5$ \\
\hline 14. Esta simulação ofereceu uma variedade de formas para avaliar a minha aprendizagem. & $4,3 \pm 0,6$ & $4,7 \pm 0,5$ & $4,5 \pm 0,5$ & $4,5 \pm 0,6$ \\
\hline \multicolumn{5}{|l|}{ Altas expectativas } \\
\hline 15. Os objetivos para a experiência simulada foram claros e de fácil compreensão. & $4,5 \pm 0,5$ & $4,7 \pm 0,5$ & $4,5 \pm 0,5$ & $4,6 \pm 0,4$ \\
\hline 16. O meu professor comunicou os objetivos e expectativas a serem alcançados durante a simulação. & $4,4 \pm 0,7$ & $4,2 \pm 0,9$ & $4,5 \pm 0,7$ & $4,3 \pm 0,7$ \\
\hline
\end{tabular}


A Tabela 4 mostra os valores médios da concordância e importância de todos os domínios presentes nas seguintes variáveis da escala de práticas educativas: aprendizagem ativa e colaboração, para cada grupo.

\section{DISCUSSÃO}

As percepções dos estudantes sobre a simulação clínica enquanto estratégia de ensino e aprendizagem foi avaliada utilizando um questionário de práticas educativas e uma escala de satisfação e autoconfiança aplicadas com um grupo controle e um grupo experimental, através da comparação das seguintes estratégias de ensino e aprendizagem: aula expositiva e simulação clínica para treino de habilidades; e aula expositiva, simulação clínica para treino de habilidades e simulação de alta fidelidade.

Os resultados apontaram para um reconhecimento da importância da utilização de estratégias de ensino diversificadas e abarque metodologias ativas de ensino nos dois grupos (controle e experimental). Entretanto, o grupo experimental apresentou maiores escores no que se refere aos domínios de práticas educativas em sete de oito domínios.

Nesse contexto, sabe-se que as práticas educacionais permeiam as metodologias e estratégias de ensino [17]. Boas práticas educacionais implicam em atender princípios que contribuam para a aprendizagem, a saber: o incentivo do contato estudante-docente, o estímulo à cooperação entre os estudantes e a aprendizagem ativa, a oferta de feedback imediato, a ênfase no tempo para estudo, o estabelecimento de altas expectativas, e o respeito aos talentos dos estudantes e suas formas de aprendizagem [18].

Para que a aprendizagem seja ativa, é indispensável que o estudante tenha a oportunidade de discutir e ampliar seus conhecimentos a partir da ativação dos conhecimentos prévios - conceitos, experiências, entre outros - e compartilhá-los junto ao facilitador, outros estudantes e colaboradores [19].

A participação ativa do estudante nos cenários simulados permite a este, pensar criticamente e analisar as diversas situações práticas. Esta reflexão atua como um dispositivo para melhorar a atuação profissional $[19,20]$. Além disso, quanto maior a oportunidade de uma experiência ativa de aprendizado, maior a habilidade que o estudante tem de assimilar as informações que são facilitadas durante o processo de aprendizagem [21,22].

Durante a simulação, é importante que os estudantes participem ativamente da sessão de feedback. Nesta etapa, oportuniza-se a reflexão e compreensão da experiência vivenciada a partir das decisões que foram tomadas. Os facilitadores devem auxiliar nesse processo a partir de comentários que pode ajudar o estudante a explorar e ou enxergar aspectos que não conseguiram identificar inicialmente e que estavam relacionados aos objetivos da aprendizagem.

Além dos momentos destinados a simulação, os tutores devem disponibilizar, em tempo oportuno, espaços para eventuais clarificação de dúvidas e orientação no sentido de guiar o estudante para atingir os objetivos da aprendizagem e a aquisição de competências e habilidades. Além de momentos junto a um grupo de estudantes, é indispensável à orientação individual.

Para aulas de exposição dialogada e simulação clínica para o treino de habilidades o docente deve reconhecer a importância da escolha correta e ou construção dos materiais e recursos didáticos a serem utilizados. Além disso, deve conferir espaço e tempo para fornecer feedback das atividades propostas. Quando optar por estratégias mais dinâmicas e que mobilize o estudante para a aprendizagem ativa, é relevante refletir sobre o preparo do estudante para lidar com a situação planejada.

Nesta pesquisa, os estudantes que participaram da formação com a estratégia de simulação clínica de alta-fidelidade perceberam e atribuíram maiores índices de concordância e importância, o que gerou maiores médias e menores desvio padrão nas variáveis do domínio aprendizagem ativa, em relação aos estudantes que participaram apenas da simulação clínica para o treino de habilidades. Neste domínio, o grupo controle apresentou os escores médios de 4,3 $\pm 0,3$ para a concordância, e 4,5 $\pm 0,4$ para a importância. Já no grupo experimental o escore médio de $4,5 \pm 0,3$ na subescala de concordância e 4,5 $\pm 0,4$ na subescala de importância.

Estudo realizado com 199 estudantes do curso de graduação em enfermagem que examinou a percepção sobre as melhores práticas educacionais em simulação e avaliou sua satisfação e autoconfiança na simulação usando simuladores de média e alta-fidelidade e paciente padronizados. O Questionário de Práticas Educacionais apresentou médias altas, com exceção da participação ativa e da oportunidade de pensar durante a sessão de debriefing [23].

No que se refere à colaboração, durante o processo de ensino e aprendizagem, é importante que o estudante tenha a oportunidade de trabalhar com os demais colegas durante as atividades propostas no currículo. A integração entre os estudantes promove a habilidade de trabalho em grupo, atitudes, e desenvolve 
outras habilidades afetivas. Além disso, a troca de saberes é relevante e indispensável na construção do conhecimento.

A simulação promove esta possibilidade na medida em que encoraja a participação do estudante, quer seja na execução direta da tarefa ou observação. Além disso, estimula a colaboração dos participantes nos apontamentos, nas observações, e nas reflexões que são construídas durante as etapas desta estratégia.

No domínio "colaboração", o grupo experimental apresentou valores médios maiores em ambas as subescalas, a saber: $4,7 \pm 0,5$ para concordância e

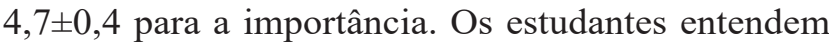
que a simulação permite uma maior possibilidade de se trabalhar com os demais colegas.

Um estudo desenvolvido no Brasil, com 37 estudantes de enfermagem, também constatou essa capacidade de integração e colaboração entre os participantes que é conferida a partir da vivência em simulações. No estudo, os autores concluem que estas capacidades auxiliam no desenvolvimento relacional e no desenvolvimento de autonomia [21].

No domínio "maneiras diferentes de aprendizagem" os estudantes do grupo 2 obtiveram maiores médias e menores desvios-padrões em todas as variáveis e subescalas. A diferença nos postos médios gerou significância estatística $(p=0,018)$. Este achado está relacionado ao caráter inovador das diferentes formas de utilizar a simulação, no sentido de proporcionar vivência prévia da prática a partir de diferentes situações que são encontradas nos serviços de assistência à saúde, em ambiente controlado [24]. Na simulação, se trabalha com habilidades que superam o domínio cognitivo. Além disso, na sessão de feedback, o estudante tem a oportunidade de avaliar sua aprendizagem.

A tecnologia de simulação deve tornar-se parte integrante do processo de ensino-aprendizagem nos currículos de enfermagem. Para tanto, é indispensável que as escolas de enfermagem criem e apoie programas de simulação e viabilizem os recursos materiais e humanos necessários para a consolidação e sucesso destes [3].

Entende-se que praticar, em um ambiente seguro, as habilidades críticas de tomada de decisões, delegação, priorização e trabalho em equipe são essenciais para o início da prática profissional de enfermagem. Sob essa perspectiva têm-se buscado identificar e fornecer evidências sobre porcentagens de substituição de práticas clínicas tradicionais por práticas que valorizam a diferentes formas de simulação [3].

As estratégias mais tradicionais, a depender do planejamento do docente, nem sempre se tem possibilidade de aproximar o estudante das realidades as quais estes serão inseridos. Além disso, podem ser incapazes de permitir o desenvolvimento de níveis cognitivos superiores, habilidades relacionais, e a eficácia no desempenho procedimentos e condutas mais complexas. Aulas expositivas e simulação clínica para o treino de habilidades, por si só, podem ser insuficientes quando se tem como público estudantes que preferem atividades mais interativas.

Um estudo identificou razões das quais os métodos tradicionais de ensino não conseguem otimizar oportunidades clínicas, a saber: o sucesso de um dia clínico estava relacionado a realização de tarefas; apenas um tipo de pedagogia foi utilizado os estudantes perderam muitas oportunidades de aprendizagem; e os estudantes cuidavam individualmente de um paciente/ usuário e o trabalho em equipe era inviabilizado [25].

Objetivos bem definidos e criatividade são os pontos de partida para o sucesso das atividades pedagógicas. Estes auxiliam o docente na condução da aprendizagem e permite ao estudante compreender o percurso metodológico adotado e o conhecimento que está sendo facilitado. Além disso, permite o docente estabelecer métodos de avaliação adequados e guiar os estudantes para a consolidação do que foi planejado [15]. No processo de definição dos objetivos, a taxonomia de Bloom pode auxiliar o docente nesta escolha e planejamento; pois orienta e fornece a estrutura para desenvolver e nivelar os objetivos para os resultados da aprendizagem [26].

Os docentes de enfermagem devem ter consciência das estratégias educacionais que adotam em suas práticas formativas. Quando se pensa em mesclar diferentes estratégias, inclusive a simulação de altafidelidade, essa consciência é um fundamento relevante para que se possa pensar na hierarquia dos cenários frente aos objetivos da aprendizagem [27].

Ao se tratar de simulação como ferramenta pedagógica, a estrutura S.M.A.R.T oferece orientações para o estabelecimento, desenvolvimento e consolidação dos objetivos da aprendizagem [15]. Quando os objetivos de aprendizagem desejados são alcançados, eles devem ser reajustados para níveis mais altos, possibilitando a aprendizagem contínua [27].

Neste estudo, os postos médios referentes à concordância e importância da dimensão "altas expectativas", foram bastante aproximadas em ambos os grupos. No grupo 1, as maiores médias foram atribuídas na subescala de concordância (média global de 4,5 $\pm 0,6$ ). Já na subescala de importância, o grupo 2 apresentou média global de $4,5 \pm 0,5$. Portanto, 
tanto as estratégias mais tradicionais (aula expositiva e simulação para o treino de habilidades) quanto à simulação de alta fidelidade são estratégias que geram altas expectativas.

Os resultados estão em consonância com a literatura. Um estudo do tipo pesquisa-ação realizado em uma universidade federal brasileira, identificou que a simulação gerou altas expectativas em $70 \%$ dos estudantes que participaram da pesquisa [24]. Um estudo com 54 estudantes de uma universidade americana que aplicou a questionário de práticas educativas no período de um ano a partir do uso de práticas simuladas referentes a conteúdos de saúde do adulto, as práticas simuladas foram bem avaliadas pelos estudantes, com escores médios entre 4 e 5 nos itens [22].

$\mathrm{Na}$ Coreia, a simulação tem sido cada vez mais utilizada em razão dos embates éticos relacionadas à vivência dos estudantes com usuário. Além disso, algumas a acreditação de universidades voltadas para a formação em enfermagem também leva em consideração a capacidade da instituição em fornecer práticas baseadas em simulação. Entretanto, ainda há dificuldades para a inserção da educação baseada em simulação nos currículos e a falta de pessoal capacitado para executar e avaliar os cenários conforme os objetivos da aprendizagem [27].

Há evidência na descrição e padronização de técnicas e estrutura para o desenvolvimento de cenários, debriefing e avaliações. Além disso, há evidências sobre a eficácia da estratégia no desenvolvimento de diversas competências e habilidades. Porém, as evidências que têm sido produzidas ao que se refere à educação baseada em simulação e currículo baseado em simulação ainda são escassas [27].

No Brasil, acompanha-se um processo de discussão sobre a necessidade da formação de profissionais de saúde que atendam as demandas do setor saúde e, sobretudo, a proposta do Sistema Único de Saúde. Além de formar o estudante para o bom funcionamento e a melhoria da qualidade prestada aos usuários, as instituições de ensino superior são desafiadas a formar profissionais que sejam capazes de transformar realidades.

Para tanto, é relevante e indispensável refletir sobre questões relacionadas com a equidade, a segurança do paciente, a ética, a bioética, os direitos humanos, o desenvolvimento do pensamento crítico e a tomada de decisão. Com isso, é preciso ampliar os horizontes no sentido de visualizar estratégias de ensino e aprendizagem que consigam contemplar, durante o processo de formação, essas perspectivas. Ao considerar os resultados apresentados e as perspectivas anteriormente apresentadas, sugere-se que a simulação de alta fidelidade realizada a partir da criatividade do docente, não atrelada aos altos custos dos centros de simulação, possa ser inserida e disseminada nos currículos de enfermagem.

\section{CONCLUSÃO}

O estudo identificou, a partir do Questionário de Práticas Educativas, que ambas as sequencias de estratégias de ensino e aprendizagem, aula expositiva dialogada, seguida de simulação para treino de habilidades e aula expositiva dialogada, seguida de simulação para treino de habilidades e simulação clínica de alta fidelidade foram avaliadas positivamente pelos estudantes. Porém, os estudantes que participaram da simulação de alta fidelidade, apresentaram médias superiores em sete dos oito domínios das subescalas de concordância e importância.

A estratégia da simulação de alta fidelidade pontua os requisitos de uma boa prática educacional na medida em que proporciona uma aprendizagem ativa, colaborativa, diferente da estratégia que proporciona a simulação apenas para o treino de habilidades. Entretanto, não se pode desconsiderar o valor das diferentes práticas pedagógicas de ensino. $\mathrm{O}$ uso de metodologias ativas para o ensino necessita ser bem estruturadas e contemplar os pressupostos da aprendizagem significativa.

Neste sentido, sugere-se que a estratégia da simulação clínica de alta fidelidade possa ser inserida no currículo na formação de enfermeiros em complemento a outras estratégias de ensino e aprendizagem. Boas práticas educacionais implicam em melhor qualificação profissional.

Considerou-se como limitação do estudo a amostra de 34 sujeitos. Além disso, os estudantes do grupo experimental tiveram a oportunidade de vivenciar uma estratégia de ensino e aprendizagem a mais que o grupo controle, o que pode ter influenciado nos escores das dimensões da escala utilizada. Porém, o estudo não objetivou aferir escores de conhecimento, e sim concepções sobre diferentes práticas educativas. Logo, as evidências produzidas poderão contribuir para preencher lacunas na literatura.

\section{NOTAS}

Parte deste trabalho é resultado de tese de doutorado em Enfermagem na Atenção à Saúde de um dos autores (RROC) intitulada "Eficácia da simulação realística no ensino de imunização de adultos no contexto da graduação em enfermagem", 2018, Centro de Ciências da Saúde, Universidade Federal do Rio Grande do Norte, Brasil. 


\section{Apoio financeiro}

O presente trabalho foi realizado com apoio da Coordenação de Aperfeiçoamento de Pessoal de Nível Superior - Brasil (CAPES).

Declaração de conflito de interesses

Os autores declaram não haver conflitos de interesses relevantes ao conteúdo deste estudo.

\section{Contribuições dos autores}

Todos os autores fizeram contribuições substanciais para concepção, ou delineamento, ou aquisição, ou análise ou inter- pretação de dados; e redação do trabalho ou revisão crítica; e aprovação final da versão para publicação.

Disponibilidade dos dados e responsabilidade pelos resultados

Todos os autores declaram ter tido total acesso aos dados obtidos e assumem completa responsabilidade pela integridade destes resultados.

\section{REFERÊNCIAS}

1. Marin MJS, Lima EFG, Paviotti AB. Aspectos das fortalezas e fragilidades no uso das metodologias ativas de aprendizagem. Rev Bras Educ Med. 2010 [cited 2018 June. 12];34(1):13-20. Available from: http://dx.doi. org/10.1590/S0100-55022010000100003

2. Costa RRO, Medeiros SM, Martins JCA, Menezes RMP, Araújo MS. O uso da simulação no contexto da educação e formação em saúde e enfermagem: uma reflexão acadêmica. Espaç. Saúde. 2015 [cited 2018 June. 12]; 16(1):59-65. Available from: http://dx.doi.org/10.22421/1517-7130.2015v16n1p59

3. Jeffries P. The Good News-Simulations Work, So Now What? J Nurs Educ. 2015 [cited 2018 June. 12]; 54(11): 603-60. Available from: https://doi.org/10.3928/01484834-20151016-10

4. Ribeiro JF, Costa JML, Silva MAC, Luz VLES, Veloso MV, Ribeiro ALL, Coelho DMM. Prática pedagógica do enfermeiro na docência do ensino superior. Rev enferm UFPE. 2018 [cited 2018 June. 12]; 12(2):291-302. Available from: https://doi.org/10.5205/1981-8963-v12i2a25129p291 -302-2018

5. Anastaciou LGC, Alves LP. Processos de Ensinagem na Universidade: pressupostos para as estratégias de trabalho em aula. 5th ed. Joinvile (SC): Univille; 2003.

6. Souza ALT, Passaglia P, Cárnio EC. Uso de simulador realístico de alta fidelidade no ensino da fisiologia humana no curso de enfermagem. Revista de Graduação USP. 2018 [cited 2019 Aug 12];3(2):113-8. Available from: https://doi.org/10.11606/issn.2525-376X.v3i 2p113-118

7. Norman G, Dore K, Grierson L. A relação mínima entre fidelidade de simulação e transferência de aprendizagem. Med Educ. 2012 [cited 2019 Aug 12]; 46(7):636-47. Available from: https://doi.org/10.1111/j.1365-2923.2012.04243.x

8. Teixeira CRS, Kusumota L, Braga FTMM, Gaioso VP, Santos CB, Silva VLS, et al. O uso de simulador no ensino de avaliação clínica em enfermagem. Texto contexto - enferm. 2011 [cited 2019 Aug 20];20(spe):187-93. Available from: http://dx.doi.org/10.1590/S01 04-07072011000500024.

9. Valadares AFM, Magro MCS. Opinião dos estudantes de enfermagem sobre a simulação realística e o estágio curricular em cenário hospitalar. Acta Paul Enferm. 2014 [cited 2018 June. 12];27(2):138-43. Available from: http://dx.doi.org/10.1590/1982-0194201400025

10. Khamis NN, Satava RM, Alnassar SA, Kern DE. A stepwise model for si mulation-based curriculum development for clinical skills, a modification of the six-step approach. Surg Endosc. 2016 [cited 2018 June. 12];30(1):279-87. Available from: https://www.ncbi.nlm.nih.gov/pubmed/25899812. https://doi.org/10.1007/s00464-015-4206-x

11. Parker RA, Mcneill J, Howard J. Comparing pediatric simulation and traditional clinical experience: Student perceptions, learning outcomes, and lessons for faculty. Clin Simul Nurs. 2015 [cited 2018 June. 12];11(3):188-93. Available from: https://doi.org/10.1016/j.ecns.2015.01.002

12. Ramm D, Thomson A, Jackson A. Learning clinical skills in the simulation suite: the lived experiences of student nurses involved in peer teaching and peer assessment. Nurse Educ Today. 2015 [cited 2018 June. 12]; 35(6):823-7. Available from: https:/www.ncbi.nlm.nih.gov/pubmed/25697946. https://doi.org/10.1016/j.nedt.2015.01.023

13. Cassiani SHB, Wilson LL, Mikael SSE, Peña LM, Grajales RAZ, McCreary LL, Theus L, Agudelo MCG, Felix AS, Uriza JM, Gutierrez NR. The situation of nursing education in Latin America and the Caribbean towards universal health. Rev Latino-Am. Enfermagem. 2017 [cited 2018 Aug 01]; 5:e2913. Available from: http://dx.doi. org/10.1590/1518-8345.2232.2913 
14. Janicas RCSV, Fernandes MGO. Como Treinar Habilidades modelos de Guias e Check-lists. In: Quilici AP, Abrão KC, Timerman S. Simulação Clínica: do conceito à aplicabilidade. São Paulo: Atheneu; 2012. p.49-76.

15. Committee IS. INACSL Standards of Best Practice: SimulationSM Simulation Design. Clin Simul Nurs. 2016; 12:13-5. https://doi.org/10.1016/j.ecns.2016.09.006

16. NLN. National League for Nursing.Simulation Innovation Resource Center. 2013 [cited 2018 June. 12]. Available from: http://sirc.nln.org/mod/glossary/view.php?id=183

17. Almeida RGS, Mazzo A, Martins JCA, Souza-Junior VD, Mendes IAC. Validação para a língua portuguesa do Educational Practices Questionnaire (Student Version). Acta paul. enferm. 2016 [cited 2019 June 26]; 29(4): 390-6. Available from: http://dx.doi.org/10.1590/1982-0194201600054.

18. Chickering AW, Gamson ZF. Seven principles for good practice in undergraduate education. AAHE Bulletin. 1987 [cited 2018 June. 12]; 39(7):3-7. Available from: www.lonestar.edu/multimedia/sevenprinciples.pdf

19. Costa RRO, Medeiros SM, Martins JCA, Dias VR. Perceptions of nursing students on the structural dimensions of clinical simulation. Sci Med. 2019 [cited 2018 June 12]; 29(1):e32972. Available from: https://doi.org/10.15448/ 1980-6108.2019.1.32972.

20. Costa RRO, Mederos SM, Martins JCA, Cossi MS, Araújo MS. Percepção de estudantes da graduação em enfermagem sobre a simulação realística. Rev Cuid. 2017 [cited 2018 June 12] 8(3):1799-808. Available from: https://doi.org/10.15649/cuidarte.v8i3.425

21. Leigh GT. High-Fidelity Patient Simulation and Nursing Students Self-Efficacy: a review of the literature. Int J Nurs Educ Scholarsh. 2008;5(1):1-16. Available from: https://doi.org/10.2202/1548-923X.1613

22. Cummings CL, Connelly LK. Can nursing students' confidence levels increase with repeated simulation activities? Nurs educ today. 2016 [cited 2018 June. 12]; 36:419-21. Available from: https://www.ncbi.nlm.nih.gov/ pubmed/26599594. https://doi.org/10.1016/j.nedt.2015.11.004

23. Zapko KA, Ferranto MLG, Blasiman R, Shelestak D. Evaluating best educational practices, student satisfaction, and self-confidence in simulation: A descriptive study. Nurs Educ Today. 2017 [cited 2018 June. 12]; 60:28-34. Available from: https://www.ncbi.nlm.nih.gov/pubmed/28987895. https://doi.org/10.1016/j.nedt.2017.09.006

24. Costa RRO. A simulação realística como estratégia de ensino-aprendizagem em enfermagem, em Natal/RN [dissertação]. Natal: Pós-graduação em Enfermagem da Universidade Federal do Rio Grande do Norte; 2014.

25. McNelis AM A, Ironsode PM, Ebright PR, Dreifuerst KT, Zvonar SE, Conner SC. Learning nursing practices: a multisite, multi-method, investigation of clinical education. J. Nurs. Regul. 2014 [cited 2018 June. 12]; 4(4): 31-5. Available from: https://www.journalofnursingregulation.com/article/S2155-8256(15)30115-0/fulltext. https://doi.org/10.1016/S2155-8256(15)30115-0

26. Bloom BS. Taxonomy of educational objectives: The classification of educational goals. Handbook 1: Cognitive domain. New York: Longman; 1956.

27. Lee JY, Lee SH, Kim JH. A review of the curriculum development process of simulation-based educational intervention studies in Korea. Nurs Educ Today. 2018 [cited 2018 June. 12]; 64:42-8. Available from: https://www. ncbi.nlm.nih.gov/pubmed/29459191. https://doi.org/10.1016/j.nedt.2018.01.029 € 